\title{
Preferential pairing in the germ line limited chromosomes of Acricotopus lucidus (Diptera, Chironomidae)
}

\author{
WOLFGANG STAIBER \\ Institut für Allgemeine Genetik, Universität Hohenheim, Garbenstrasse 30, D-7000 Stuttgart 70, FRG
}

\begin{abstract}
With G-banding and X-ray induced marker chromosomes, pairing preferences of identical over homologous germ line limited chromosomes were demonstrated in male meiosis of Acricotopus lucidus. The consequences of preferential identical pairing are discussed in respect to genetic recombination within the germ line limited chromosomes.
\end{abstract}

Keywords: Acricotopus lucidus, germ line limited chromosomes, preferential pairing.

\section{Introduction}

Chromosomes limited to the germ line and eliminated from the future soma cells during early embryonic development were found in Acricotopus lucidus (Chironomidae, Orthocladiinae, Bauer \& Beermann, 1952). These germ line limited chromosomes (=Ks, K derived from 'Keimbahn', Bauer, 1970) and the soma chromosomes $(=\mathrm{Ss})$ pass through a complex chromosome cycle which exhibits three remarkable specialities (for review, see Beermann, 1956; White, 1973):

1 The complete elimination of the $\mathrm{Ks}$ from the prospective somatic cells during the early cleavage divisions (soma elimination).

2 The elimination of about half of the Ks during the first gonial mitoses of the primordial germ cells in newly hatched larvae (germ line elimination, Bauer \& Beermann, 1952).

3 A compensating duplication of the $\mathrm{Ks}$ as a result of the last gonial mitosis in the young fourth instar larvae. In this so-called differential mitosis all $\mathrm{Ks}$ move undivided to one cell pole, whereas the Ss behave as in a normal mitosis. The cells with Ss and Ks develop into regular spermatocytes and oocytes, but the cells with the $\mathrm{S}$ set only differentiate into aberrant spermatocytes or into nurse cells.

The knowledge of the function of the Ks is still very restricted (for a review, see Hennig, 1986; Tobler, 1986). In the Cecidomyiid Wachtliella persicariae, the $\mathrm{Ks}$ are indispensable for normal development of germ cells in both sexes (Geyer-Duszyńska, 1966).

$\mathrm{G}$-banding permits the differentiation of nine $\mathrm{K}$ types, $\mathrm{K} 1-\mathrm{K} 9$, in $A$. lucidus, which in various numbers and combinations form the gonial $\mathrm{K}$ complements (Staiber, 1988). In gonial metaphases each K type may be present once or twice, some Ks are also present on three occasions, and a special $\mathrm{K}$ type, $\mathrm{K} 4$, up to five times. A K chromosome present only once before duplication in differential mitosis (see point 3 above) forms a bivalent in meiosis with its identical copy, an autobivalent. If we assume free meiotic pairing, a chromosome of the $\mathrm{K}$ type, which is present two or more times in gonial cells, can form either an autobivalent, a bivalent with one of its homologues, or multivalents together with its identical copy and its homologues (Staiber, 1989).

In addition to random chromosome pairing in bivalents, pairing preferences are also possible, i.e. identical over homologous chromosomes, as found in studies of meiosis of spontaneous tetraploid testes cells in the grasshopper Euchorthippus pulvinatus (Giraldez \& Santos, 1981; Santos et al., 1983).

In order to see whether there are also pairing preferences in the Ks of $A$. lucidus, an attempt was made to obtain marker Ks with cytologically visible chromosome mutations by $\mathrm{X}$-raying male imagines. These could be used, together with G-banding, to distinguish identical and homologous chromosomes in meiosis of $\mathrm{F}_{1}$-larvae.

\section{Materials and methods}

$\mathrm{X}$-irradiation of male imagines of Acricotopus lucidus was carried out with a Philips X-ray machine 'Macrotank G $300^{\prime}(200 \mathrm{kV}, 5 \mathrm{~mA}$, filtration $0.3 \mathrm{~mm} \mathrm{Cu}$ and 3 $\mathrm{mm} \mathrm{Al}$, half-value layer $11 \mathrm{~mm} \mathrm{Al}$, focus distance 
$15 \mathrm{~cm}$, equal to about $160 \mathrm{R} \mathrm{min}^{-1}$, exposure $17 \mathrm{~min}$ ). The dose was not measured. Males were then placed together with non-irradiated females.

Testes with meiotic cells were obtained from $\mathrm{F}_{1}$ larvae in transitional stages from the fourth larval instar to prepupa (Staiber \& Behnke, 1985). Hypotonic treatment of gonads, slide preparation and G-banding followed the methods described in Staiber (1988).

\section{Results and discussion}

In this study, G-banded meiosis preparations of 310 male $\mathrm{F}_{1}$-larvae were screened for $\mathrm{X}$-ray induced cytological visible $\mathrm{K}$ chromosome mutations. Only metaphases I were examined. In $A$. lucidus the number of $\mathrm{K}$ bivalents ranged from 6 to 16 (Staiber, 1988). It is probable that only a proportion of all Ks with chromosome alterations was detected, because only mutated $\mathrm{Ks}$ with conspicuous changes in the positions of centromeres, or of characteristic G-bands, or with alterations to the total length of chromosomes or chromosome arms, could be recognized as such. Furthermore, some preparations contained no metaphase I stages, while in others with metaphase I cells, the quality of G-banding which resulted was insufficient to allow chromosome analysis.

Ks with cytologically visible chromosome mutations were found in 29 larvae. In addition, 58 of the 310 larvae exhibited chromosome mutations in the giant salivary gland chromosomes, representing the somatically paired and polytenized Ss (in A. lucidus $n=3$ ). The following Ks were seen to be affected by deletions, inversions or translocations: $\mathrm{K} 1, \mathrm{~K} 2, \mathrm{~K} 4, \mathrm{~K} 6$ and $\mathrm{K} 8$. Some are presented in Fig. 1a,b and Fig. 2a-d. Most of the mutations were found on the largest $\mathrm{K}, \mathrm{K} 1$, characterized by a conspicuous pattern of thick dark bands that facilitate recognition of the alterations.

Each testis of a newly hatched $A$. lucidus larva contains only one primary germ cell (Bauer \& Beermann, 1952). During the first gonial mitoses of these cells about half of the Ks are eliminated by lagging. It is not known whether paternal or maternal Ks are eliminated equally or preferentially. The occurrence of X-ray induced marker $\mathrm{Ks}$ excludes the possibility that all paternal Ks will be eliminated in the first gonial mitoses in males.

In one of the $F_{1}$ larvae, two different marker Ks were found in one testis, while in the other testis only one of the marker Ks was present. This corroborates earlier observations (Staiber, 1988) that, during germ line elimination in both testes of a young first instar larva, it is not always the same Ks that are eliminated.

In only nine of the 29 larvae marker Ks, together with two or four normal homologous Ks, were present.

Table 1 Frequencies of identical and homologous pairing of germ line limited chromosomes in male meiosis of Acricotopus lucidus

\begin{tabular}{|c|c|c|c|c|c|}
\hline \multirow{2}{*}{$\begin{array}{l}\text { Animal } \\
\text { number }\end{array}$} & \multirow{2}{*}{$\begin{array}{l}\mathrm{K} \text { combination } \\
\text { analysed }\end{array}$} & \multirow{2}{*}{$\begin{array}{l}\text { Number of } \\
\text { cells }\end{array}$} & \multicolumn{2}{|c|}{$\begin{array}{l}\text { Only } \\
\text { bivalents }\end{array}$} & \multirow[b]{2}{*}{ Other configurations } \\
\hline & & & I & $\mathbf{H}$ & \\
\hline 1 & Inv.K1, K1 & 115 & 114 & 1 & - \\
\hline 2 & Transl.K1, K1, K1 & 24 & 22 & - & $2(\operatorname{IVmK} 1 / \mathrm{K} 1+\mathrm{IIK} 1)$ \\
\hline 3 & Del.K1, K1 & 37 & 36 & 1 & - \\
\hline 4 & Transl.K1, K1 & 41 & 41 & - & - \\
\hline 5 & Inv.K1, K1 & 22 & 22 & - & - \\
\hline 6 & Inv.K1, K1 & 41 & 40 & - & $1(\mathrm{IVmK} 1 / \mathrm{K} 1)$ \\
\hline 7 & Del.K4, K4, K4 & 43 & 39 & - & $\begin{array}{l}2(\mathrm{IVmK} 4 / \mathrm{K} 4+\mathrm{IIK} 4) \\
1(\mathrm{IIK} 4+\mathrm{IK} 4+\operatorname{IImK} 4) \\
1(2 \mathrm{IIK} 4+2 \operatorname{ImK} 4)\end{array}$ \\
\hline 8 & Del.K6, K6 & 23 & 20 & - & $\begin{array}{l}1(\mathrm{IVmK} 6 / \mathrm{K} 6) \\
1\left(\mathrm{IVK} 2 / \mathrm{K} 6^{*}+\operatorname{ImK} 6\right) \\
1\left(\mathrm{VK} 2 / \mathrm{mK} 6 / \mathrm{K} 6^{*}+\operatorname{ImK} 6\right)\end{array}$ \\
\hline 9 & Del.K8, K8 & 20 & 18 & 1 & $1(\mathrm{IVmK} 8 / \mathrm{K} 8)$ \\
\hline
\end{tabular}

$\mathrm{I}=$ Cells with identical pairing.

$\mathrm{H}=$ Cells with homologous pairing.

Del.K, Inv.K, Transl.K: Marker K type with deletion, inversion or translocation.

$\mathrm{mK}=\mathrm{K}$ type with chromosome mutation = marker $\mathrm{K}$ type.

I, II, III, IV, V: Uni-, bi-, tri-, quadri-, quinquivalent.

*Multivalent composed of non-homologous Ks. 


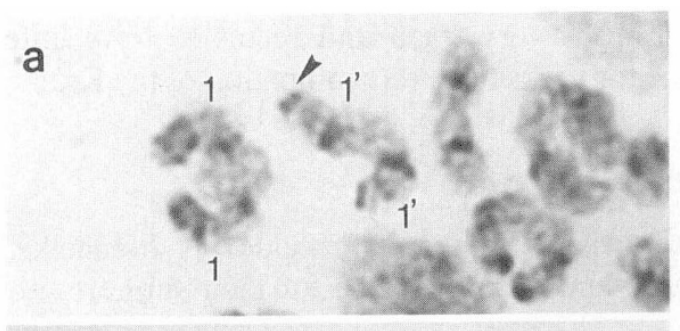

b

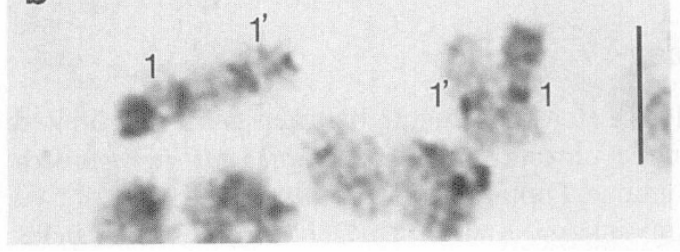

Fig. 1 Sections of G-banded metaphases I of animal no. 3 showing normal K1s (=1) and X-ray induced marker K1s $\left(=1^{\prime}\right)$ with a deletion of the terminal part of the long arm (arrowhead). Two thick dark G-bands are lost. Two bivalents formed by pairing of identical chromosomes (a) and two bivalents formed by pairing of homologous chromosomes (b). Bar represents $5 \mu \mathrm{m}$.

This allowed pairing analysis, the results of which are presented in Table 1 . No random pairing between identical and homologous $\mathrm{Ks}$ was found. The results show a large excess of identical associations. Homologous pairing in bivalents seems to be rather an exception. Of all metaphase I cells containing marker Ks together with two or four homologous Ks, 352 showed identical chromosome pairing, and only three showed pairings of marker Ks and homologous Ks (see animals nos 1, 3, 9; Fig. 1b). Another seven metaphase I cells exhibited quadrivalents composed of marker Ks and homologous Ks, and four metaphase I cells showed still other configurations of marker $\mathrm{Ks}$, homologous and non-homologous Ks.

The preferential pairing of identical Ks might be explained by their closer proximity and therefore higher pairing affinity among identical chromosomes, as suggested by Benavente \& Orellana (1989). In $A$. lucidus the doubling of the Ks resulted from the last unequal gonial mitosis. Under the conditions used to rear $A$. lucidus larvae, the time between differential mitosis and the time meiotic cells reach metaphase $\mathrm{I}$ is about $150 \mathrm{~h}$. In differential mitosis both sister chromatids of a $\mathrm{K}$ move undivided to one cell pole. It is not known whether later the identical Ks remain associated or spatially arranged until meiosis. If so, this might explain the excess of identical pairing detected. Santos et al. (1983), who found preferential identical over homologous pairing in spontaneous tetraploid grasshopper spermatocytes, also could not exclude the possibility of premeiotic chromosome associations. In one case the primary spontaneous polyploid cell underwent at least seven gonial mitoses prior to entry into meiosis.

In $A$. lucidus there is a clear tendency towards the spatial association of homologous $\mathrm{Ks}$ in gonial mitoses (Fig. 2a-c, in Staiber, 1988) and of homologous K bivalents in meiosis (Fig. 1 in Staiber, 1989). The latter was confirmed again in the present study. For animal no. 3 in Table 1, for example, in about three-quarters of all metaphases I examined marker K1 bivalents and $\mathrm{K} 1$ bivalents lie next to each other (see Fig. 1a).

It cannot be excluded that the chromosome mutations, which indicate the marker Ks, have an influence on pairing behaviour, e.g. a terminal deletion, as in Fig. $1 \mathrm{a}$, may lead to a loss of zygomeres (proposed functional units of chromosome pairing; Sybenga, 1966), which would then change the pairing activity of the marker chromosome. But also on marker Ks without deleted parts [e.g. with a small inversion (animal no. 5, Fig. 2a) or an additional small translocated chromosome part (animal no. 2, Fig. 2b)] preferential identical pairing was found to the same extent. In the present study the effects of the chromosome mutations on pairing cannot be independently assessed or separated from other factors which might operate to bring about
Fig. 2 Sections of G-banded metaphases I with marker Ks $\left(^{\prime}\right)$. (a) Animal no. 5. Two bivalents K1 formed by identical pairing. Arrowhead indicates a small inversion. (b) Animal no. 2. Quadrivalent composed of normal and marker K1s. Arrowhead indicates a translocated chromosome part. (c) Animal no. 7. Three bivalents of $\mathrm{K} 4 \mathrm{~s}$ and marker $\mathrm{K} 4 \mathrm{~s}$ formed by identical pairing. Arrowhead indicates deletion. (d) Animal no. 7. Chain quadrivalent composed of normal and marker K4s. Bar represents $5 \mu \mathrm{m}$.

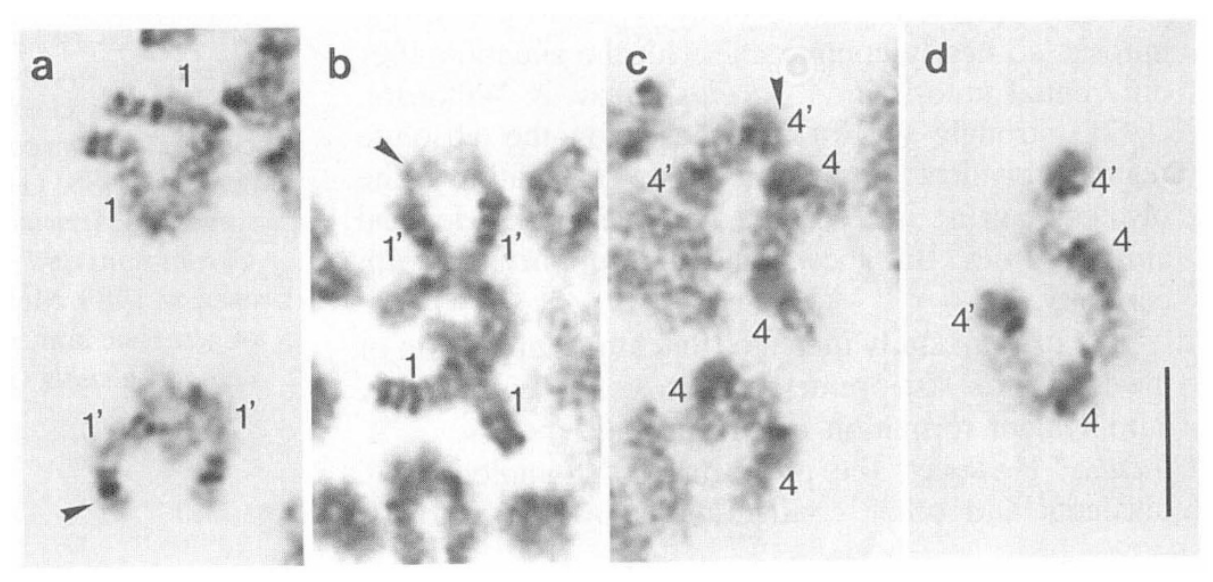


preferential pairing. However, there are two aspects of the pairing data presented which can be interpreted to indicate a strong tendency towards preferential identical pairing, which is independent of the effects of chromosome structural mutation. (a) There is a very high overall proportion of identical pairing. This suggests that factors other than structural chromosome differentiation operate as structural effects alone might not be expected to lead to almost exclusively identical pairing. (b) There is very little variation in the degree of preferential identical pairing between males carrying different structural chromosome mutations, such as might be expected if chromosome mutations were the principal factor causing preferential pairing.

Most of the $\mathrm{K}$ bivalents show one or two chiasmata. In a few bivalents the Ks are paired but not associated by chiasmata. From equal crossing over between identicals in autobivalents, no genetic recombination within a $\mathrm{K}$ type is to be expected. As seen in Table 1 in this study and in table 1 in Staiber (1989), exchanges between homologous Ks by multivalent formation (Fig. $2 \mathrm{~b}$ and d, eight cases in Table 1) seem to play a more important role for genetic recombination within the $\mathrm{K}$ types in $A$. lucidus than do exchanges in the rare bivalents composed of homologous Ks. In one animal (no. 8 in Table 1) two multivalents, a quadrivalent and a quinquivalent, composed of non-homologous Ks participating with marker Ks or their homologues were found. In a recent report (Staiber, 1989), multivalent formation and crossing over between non-homologous Ks were discussed in connection with the evolution of the $\mathrm{K}$ type diversity in $A$. lucidus.

Complete autobivalent formation in oocytes and some of the spermatocytes was reported for the stick insect Carausius morosus (Koch et al., 1972; Pijnacker \& Koch, 1975). The autobivalent formations present, however, resulted from an extra chromosome duplication step in early meiotic prophase leading to a 'tetrapachytene' stage with a doubled amount of DNA. In the grasshopper, Caledia captiva, where tetraploidy probably occurred spontaneously in the last premeiotic mitosis (so nearly comparable with the situation after differential mitosis in $A$. lucidus), Shaw \& Wilkinson (1978) strongly suggest that, based on the 'absolute regular bivalent formation and segregation', the bivalent pairing is restricted to molecularly identical chromosomes. But they could not support this with certainty.

The present study indicates that after duplication of the $\mathrm{Ks}$ in the last premeiotic mitosis, no complete $\mathrm{K}$ autobivalent formation occurs in male meiosis of $A$. lucidus. However, the proportion of homologous $\mathrm{K}$ bivalents and other configurations involving homo- logous pairing is very small and seems to have little importance for the genetic recombination of the Ks.

\section{Acknowledgements}

The author wishes to thank Professor F. Mechelke, Brigitta Aich and Irmgard Wech for their support and help.

\section{References}

BAUER, H. 1970. Rearrangements between germ-line limited and somatic chromosomes in Smittia parthenogenetica (Chironomidae, Diptera). Chromosoma, 32, 1-10.

BAUER, H. AND BEERMANN, w. 1952. Der Chromosomencyclus der Orthocladiinen (Nematocera, Diptera). Z. Naturforsch., 7b, 557-563.

BEERMANN, w. 1956. Nuclear differentiation and functional morphology of chromosomes. Cold Spring Harbor Symp. Quant. Biol., 21, 217-230.

BenaVente, E. AND orellana, J. 1989. Pairing competition between metacentric and telocentric chromosomes in autotetraploid rye. Heredity, 62, 327-334.

GEYER-DUSZYŃSKA, I. 1966. Genetic factors in oögenesis and spermatogenesis in Cecidomyiidae. Chromosomes Today, 1, 174-178.

GIRALDEZ, R. AND SANTOS, J. L. 1981. Cytological evidence for preferences of identical over homologous but not-identical meiotic pairing. Chromosoma, 82, 447-451.

HENNIG, w. 1986. Heterochromatin and germ line-restricted DNA. In: Hennig, W. (ed.) Results and Problems in Cell Differentiation, Vol. 13, Germ Line - Soma Differentiation. Springer-Verlag, Berlin, pp. 175-192.

KOCH, P., PIJNACKER, L. P. AND KREKE, J. 1972. DNA reduplication during meiotic prophase in the oocytes of Carausius morosus Br. (Insecta, Cheleutoptera). Chromosoma, 36, 313-321.

PIJNACKER, L. P. AND KOCH, P. 1975. Complete and incomplete extra DNA reduplication during spermatogenesis of Carausius morosus Br. (Insecta, Phasmida). Chromosoma, 49, 269-278.

SANTOS, J. L., ORellana, J. AND GIRAldez, R. 1983. Pairing competition between identical and homologous chromosomes in rye and grasshoppers. Genetics, 104, 677-684.

SHAW, D. D. AND WILKINSON, P. 1978. 'Homologies' between nonhomologous chromosomes in the grasshopper Caledia captiva. Chromosoma, 68, 241-259.

STAIBER, w. 1988. G-banding of germ line limited chromosomes in Acricotopus lucidus (Diptera, Chironomidae). Chromosoma, 97, 231-234.

STAIBER, w. 1989. Multivalent formation and pairing behavior of germ line limited chromosomes in male meiosis of $\mathrm{Acri}$ cotopus lucidus (Diptera, Chironomidae). Genome, 32, 941-945. 
STAIBER, W. AND BEHNKE, E. 1985. Developmental puffing activity in the salivary gland and Malpighian tubule chromosomes of Acricotopus lucidus (Diptera, Chironomidae). Chromosoma, 93, 1-16.

SYBENGA, J. 1966. The zygomere as hypothetical unit of chromosome pairing initiation. Genetica, 37, 186-198.
TOBLER, H. 1986. The differentiation of germ and somatic cell lines in nematodes. In: Hennig, W. (ed.) Results and Problems in Cell Differentiation, Vol. 13, Germ Line Soma Differentiation. Springer-Verlag: Berlin, pp. 1-69. whITE, M. J. D. (1973) Animal Cytology and Evolution, 3rd edn, Cambridge University Press: London. 